\title{
Prevalência de sobrepeso e obesidade geral e central em mulheres idosas da cidade de Curitiba, Paraná
}

\author{
Prevalence of overweight, general and central \\ obesity in elderly women from \\ Curitiba, Paraná, Brazil
}

Cosme Franklim BUZZACHERA ${ }^{1}$

Maressa Priscila KRAUSE ${ }^{1}$

Hassan Mohamed ELSANGEDY ${ }^{1}$

Tatiane HALLAGE' ${ }^{1}$

Priscila GRANATO'

Kleverton KRINSKI ${ }^{1}$

Wagner de CAMPOS ${ }^{1}$

Sérgio Gregório da SILVA ${ }^{1}$

R E S U M O

\section{Objetivo}

Investigar a prevalência de sobrepeso e obesidade geral e central em uma amostra representativa da população de mulheres idosas do município de Curitiba, Paraná.

\section{Métodos}

Este estudo apresentou delineamento observacional, transversal e descritivo. As variáveis índice de massa corporal e da circunferência de cintura foram obtidas para a determinação do sobrepeso e obesidade geral e central, respectivamente. Medidas de tendência central, variabilidade, freqüências relativas e absolutas em cada faixa etária, dentro das categorias de índice de massa corporal e circunferência de cintura, foram verificadas.

\section{Resultados}

Participaram deste estudo 1069 mulheres com idade superior a 60 anos (média=69,5, desvio-padrão=6,1 anos). Em relação às categorias do índice de massa corporal, a maioria dos sujeitos teve seu estado nutricional classificado como sobrepeso (43,5\%), seguido por obesidade (34,0\%) e normalidade (22,5\%). Por sua vez, a obesidade $(45,1 \%)$ foi a condição nutricional prevalente em relação às categorias para a circunferência de

1 Universidade Federal do Paraná, Centro de Pesquisa em Exercício e Esporte, Departamento de Educação Física. R. Coração de Maria, 92, Jardim Botânico, 80215-370, Curitiba, PR, Brasil. Correspondência para/Correspondence to: S.G. SILVA. E-mail: <sergio.gregorio@ufpr.br>. 
526 | C.F. BUZZACHERA et al.

cintura, seguida por sobrepeso $(33,1 \%)$ e normalidade $(21,8 \%)$. Além disso, o sobrepeso e a obesidade geral diminuíram da primeira para a última faixa etária (-20,4\% e -11,6\%, respectivamente), enquanto a normalidade elevou-se substancialmente (+75,3\%). Resultados similares foram verificados em relação à adiposidade central, nos quais as condições de sobrepeso e obesidade declinaram 14,3\% e 8,3\%, respectivamente.

\section{Conclusão}

Uma elevada prevalência de sobrepeso e obesidade geral e central foi verificada entre as mulheres idosas da cidade de Curitiba (PR), independentemente da idade. Programas públicos visando à prevenção e à redução do excesso de adiposidade corporal a partir de intervenção nos padrões de ingestão dietética e gasto energético, como a prática regular de exercício físico, tornam-se necessários nesta população.

Termos de indexação: Adiposidade. Envelhecimento. Fatores socioeconômicos. Mulheres. Obesidade.

\section{A B S T R A C T}

\section{Objective}

The objective of this study was to investigate the prevalence of general and central overweight and obesity in a representative sample of the population of elderly women living in Curitiba, Paraná.

\section{Methods}

The experimental design of this study was observational, cross-sectional and descriptive. The variables body mass index and waist circumference were obtained to determine general and central overweight and obesity, respectively. Measurements of central tendency, variability, relative and absolute frequencies in each age group within the body mass index and waist circumference categories were verified.

\section{Results}

The sample consisted of 1069 women aged above 60 years (mean=69.5; standard deviation=6.1 years). In relation to body mass index, most of the subjects were classified as overweight (43.5\%), followed by obese (34.0\%) and normal (22.5\%). On the other hand, obesity (45.1\%) prevailed in relation to the categories for waist circumference, followed by overweight (33.1\%) and normal (21.8\%). Furthermore, general overweight and obesity decreased from the first to the last age group $(-20.4 \%$ and $-11.6 \%$, respectively), while normal weight increased substantially (+75.3\%). Similar results were verified in relation to central adiposity, where overweight and obesity decreased $14.3 \%$ and $8.3 \%$, respectively.

\section{Conclusion}

An elevated prevalence of general and central overweight and obesity was verified among elderly women from Curitiba, Paraná, Brazil, regardless of age. Public health programs that aim to prevent and reduce excess body fat by intervening on dietary intake patterns and energy expenditure by recommending regular physical activity are necessary for this population.

Indexing terms: Adiposity. Aging. Socioeconomic factors. Women. Obesity.

\section{N T R O D U Ç Ã O}

O aumento da obesidade tem sido considerado um importante problema de saúde pública, atingindo tanto países desenvolvidos como países em desenvolvimento ${ }^{1,2}$. No Brasil, estudos epidemiológicos transversais demonstraram que a prevalência de obesidade elevou-se, entre as décadas de 70 e 90 , de 2,4\% para 6,9\% entre os homens e de $7,0 \%$ para $12,5 \%$ entre as mulheres ${ }^{3}$. As rápidas modificações ambientais, culturais, econômicas e demográficas ocorridas nas últimas décadas na sociedade brasileira poderiam ter resultado em mudanças nos padrões de ingestão dietética e gasto energético, indicando assim um possível meio para a explicação desse processo de transição nutricional ${ }^{4}$.

As elevadas prevalências de sobrepeso e obesidade também foram observadas na população idosa brasileira. Em estudo realizado por Tavares \& Anjos ${ }^{5}$, relativo à Pesquisa Nacional sobre Saúde e Nutrição de 1989, aproximadamente metade $(50,2 \%)$ das mulheres idosas 
avaliadas apresentaram sobrepeso (índice de massa corporal - IMC $\geq 25 \mathrm{~kg} / \mathrm{m}^{2}$ ), enquanto entre os homens idosos, a prevalência foi de $30,4 \%$. Santos \& Sichieri ${ }^{6}$ apresentaram resultados similares em seu estudo envolvendo 699 sujeitos idosos moradores do município do Rio de Janeiro, no qual a prevalência de sobrepeso foi de $52,4 \%$ e $46,5 \%$ entre mulheres e homens, respectivamente. Esses resultados demonstraram uma necessidade de políticas de saúde pública que preconizem a prevenção, o controle e/ou a redução do excesso de adiposidade corporal e suas co-morbidades associadas também entre os indivíduos idosos, haja vista que esse é o segmento populacional que apresentou o maior crescimento nas últimas décadas?

Devido à sua fácil aplicabilidade e ao baixo custo operacional, o IMC tem sido utilizado como um indicador de sobrepeso (IMC $\left.\geq 25 \mathrm{~kg} / \mathrm{m}^{2}\right)$ e obesidade $\left(I M C \geq 30 \mathrm{~kg} / \mathrm{m}^{2}\right)$ geral em diversos estudos epidemiológicos envolvendo adultos e idosos ${ }^{3-6,8-10}$. Entretanto, inúmeras modificações na composição corporal são observadas com o enveIhecimento, e a utilização conjunta do IMC e de um indicador de adiposidade central para a população idosa tem sido recentemente proposta ${ }^{11-13}$. Associada positivamente ao conteúdo de tecido adiposo visceral ${ }^{13}$, a circunferência de cintura (CC) poderia ser um importante indicador de adiposidade central entre os idosos, principalmente devido à sua relação direta com o risco para morbidades e mortalidade por todas as causas e/ou doenças cardiovasculares ${ }^{14-16}$. Contudo, poucos estudos no Brasil buscaram investigar a prevalência de sobrepeso e obesidade central nessa população6,17.

Portanto, o objetivo deste estudo foi estimar a prevalência de sobrepeso e obesidade geral e central em uma amostra representativa da população de mulheres idosas do município de Curitiba (PR).

\section{MÉTO D O S}

Este estudo apresentou delineamento observacional, transversal e descritivo e foi desenvolvido no município de Curitiba (PR), no ano de 2006, como parte integrante do Projeto Terceira Idade Independente (Centro de Pesquisa em Exercício e Esporte - Universidade Federal do Paraná). Com o intuito de realizar uma seleção amostral estratificada, as seguintes etapas foram conduzidas: (1) cadastro geral dos grupos comunitários existentes no município de Curitiba, obtido mediante parceria estabelecida com instituições promotoras de atividades recreacionais para a população idosa; (2) mapeamento de todos os grupos comunitários cadastrados nas oito regionais geográficas do município; (3) alocação aleatória simples dos grupos comunitários que seriam convidados a participar da pesquisa, por regional geográfica; (4) visita ao grupo comunitário, explicação dos procedimentos da pesquisa, e convite à participação voluntária no estudo para os indivíduos pertencentes ao respectivo grupo. O processo de seleção amostral adotado no presente estudo tem sido abordado com maiores detalhes em prévias investigações ${ }^{14,18,19}$.

Participaram do estudo 1069 sujeitos do sexo feminino, todos com idade cronológica superior a 60 anos. Para a devida análise das variáveis investigadas, os participantes foram alocados em uma das cinco faixas etárias de acordo com a sua idade: 60-64 anos ( $n=296)$, 65-69 anos ( $n=308), 70-74$ anos ( $n=248), 75-79$ anos $(n=148)$ e $\geq 80$ anos ( $n=69)$. De acordo com Reijneveld ${ }^{20}$, a utilização de faixas etárias de meiadécada parece ser ideal em estudos epidemiológicos, pois evita a possibilidade de influência da idade sobre as variáveis investigadas dentro de um mesmo grupo etário.

Após detalhado esclarecimento sobre os propósitos desta investigação, procedimentos utilizados, benefícios e possíveis riscos atrelados, os sujeitos assinaram o termo de consentimento, condicionando sua participação de modo voluntário. O protocolo de pesquisa foi aprovado pelo Comitê de Ética do Setor de Ciências Biológicas da Universidade Federal do Paraná, conforme as normas estabelecidas na Declaração de Helsinki e na Resolução 196/96 do Conselho Nacional de 
Saúde sobre pesquisa envolvendo seres humanos (protocolo $n^{\circ}$ 003-06).

Com o objetivo de evitar a influência de variações cicardianas, todas as avaliações foram realizadas em um mesmo período do dia, entre 8 e 10 horas. Além disso, os participantes foram instruídos a não realizar atividade física vigorosa no dia anterior, como também a não ingerir alimento por um período de duas horas antecedentes ao seu início. Todas as avaliações foram realizadas no Laboratório de Fisiologia do Centro de Pesquisa em Exercício e Esporte, da Universidade Federal do Paraná.

As medidas antropométricas, massa corporal $(\mathrm{kg})$ e estatura $(\mathrm{cm})$ foram obtidas conforme procedimentos propostos por Lohman et al. ${ }^{21}$. Como instrumento de determinação da massa corporal foi utilizada balança digital, marca Toledo, modelo 2096 PP (precisão de 0,1 kg), enquanto que para a estatura foi utilizado estadiômetro, marca Sanny, modelo Standard, (precisão de $0,1 \mathrm{~cm}$ ), o qual encontrava-se fixado à parede. Preconizando evitar as variações inter-avaliadores, todas as medidas foram realizadas por um único avaliador previamente treinado.

O índice de massa corporal foi obtido pelo quociente massa corporal/estatura ${ }^{2}$, cujo valor foi expresso em $\mathrm{kg} / \mathrm{m}^{2}$. Os pontos de coorte selecionados para a análise do IMC foram: normalidade $\left(18,5-24,9 \mathrm{~kg} / \mathrm{m}^{2}\right)$, sobrepeso $\left(25,0-29,9 \mathrm{~kg} / \mathrm{m}^{2}\right)$ e obesidade geral $\left(\geq 30,0 \mathrm{~kg} / \mathrm{m}^{2}\right)$, conforme estabelecido pela Organização Mundial de Saúde ${ }^{11}$.

A circunferência de cintura (CC) foi obtida com a utilização de fita antropométrica ineslática (precisão de $0,1 \mathrm{~cm}$ ), conforme procedimentos propostos por Lohman et al. ${ }^{21}$. Os pontos de coorte selecionados para a análise da CC foram: normalidade $(<80 \mathrm{~cm})$, sobrepeso $(\geq 80 \mathrm{~cm}$ à $\leq 88 \mathrm{~cm})$ e obesidade $(>88 \mathrm{~cm})$ central, conforme sugerido por Lean et al. ${ }^{22}$.

O nível socioeconômico foi determinado a partir de inquérito com o avaliado, mediante utilização de questionário estruturado envolvendo o Critério de Classificação Sócio-Econômico da
Associação Nacional de Empresas de Pesquisa23. Inicialmente, segundo um sistema de pontuação, a seguinte classificação foi estabelecida: 0-5 (classe E), 6-10 (classe D), 11-16 (classe C), 17-20 (classe B2), 21-24 (classe B1), 25-29 (classe A2) e 30-34 (classe A1) pontos. Posteriormente, três categorias socieconômicas foram compostas: Alta ( 11 e A2), Média (B1 e B2) e Baixa (C, D e E).

Medidas de tendência central e de variabilidade foram aplicadas para a determinação dos valores descritivos. As freqüências relativas e absolutas das faixas etárias e do nível socioeconômico dentro das categorias de IMC e CC foram verificadas. A análise dos dados foi realizada mediante a utilização do Statistical Package for the Social Sciences (SPSS, versão 13.0) for Windows.

\section{RESULTADOS}

A Tabela 1 apresenta as medidas de tendência central e de variabilidade das variáveis massa corporal $(\mathrm{kg})$, estatura $(\mathrm{cm}), \mathrm{IMC}\left(\mathrm{kg} / \mathrm{m}^{2}\right)$, CC (cm), nível socioeconômico (pontos) e idade (anos) dos participantes do estudo. Os valores médios de IMC e CC indicaram as condições de sobrepeso geral e central, respectivamente. Em relação ao nível socioeconômico, os valores médios encontrados são representativos da categoria baixa (classe C).

Em relação ao IMC, a maioria dos sujeitos foi classificada no estado nutricional de sobrepeso $(43,5 \%)$, seguida por obesidade $(34,0 \%)$ e normalidade (22,5\%). Por outro lado, a obesidade $(45,1 \%)$ foi a condição prevalente quanto à CC, seguida por sobrepeso $(33,1 \%)$ e normalidade $(21,8 \%)$, conforme o apresentado na Tabela 2. Quando os grupos foram divididos por faixa etária, uma maior prevalência de sobrepeso (49,0\%) foi verificada no grupo de 60-64,9 anos baseando-se no IMC, e a maior parcela dos sujeitos classificados com estado nutricional de sobrepeso (37,0\%), avaliado pela CC, foi no grupo de 75-79,9 anos. Em relação à obesidade mensurada pelo IMC, a maior prevalência (37,3\%) foi observada no grupo de 65-69,9 anos, e pelo critério de CC, no grupo de $70-74,9$ anos (46,2\%). 
A Tabela 3 apresenta as freqüências relativa e absoluta das condições de normalidade, sobrepeso e obesidade geral e central, segundo nível socioeconômico. A maioria dos sujeitos foi classificada com nível socioeconômico baixo (806 sujeitos) em ambas classificações do estado nutricional (IMC e CC). O sobrepeso geral e a obesidade central foram as condições nutricionais mais prevalentes relativas ao IMC e à CC, respectivamente, contudo, o critério de CC classificou uma maior parcela dos sujeitos com estado nutricional de obesidade, comparado ao critério de IMC,

Tabela 1. Distribuição de mulheres idosas, segundo idade, variáveis antropométricas e socioeconômicas ( $n=1069)$. Curitiba (PR), 2006.

\begin{tabular}{lrrrr}
\hline Variáveis & \multicolumn{1}{c}{ M } & \multicolumn{1}{c}{ DP } & Mínimo & Máximo \\
\hline Idade (anos) & 69,5 & 6,1 & 60,0 & 88,8 \\
Massa corporal $(\mathrm{kg})$ & 68,3 & 12,3 & 39,0 & 132,1 \\
Estatura (cm) & 154,7 & 6,2 & 132,0 & 180,0 \\
IMC (kg/m²) & 28,5 & 4,7 & 17,0 & 51,6 \\
CC (cm) & 87,1 & 10,4 & 61,0 & 135,0 \\
NSE (pontos) & 13,4 & 4,6 & 0,0 & 29,0 \\
\hline
\end{tabular}

IMC: Indice de massa corporal; CC: circunferência de cintura; NSE: nível socioeconômico; M: média; DP: desvio-padrão. independentemente da categoria socioeconômica.

As prevalências de sobrepeso e obesidade geral e central em mulheres idosas, divididas por faixas etárias, são apresentadas na Figura 1. 0 sobrepeso e a obesidade geral diminuíram com o avanço da idade $(-20,40 \%$ e $-11,60 \%$, respectivamente), enquanto a normalidade elevou-se substancialmente $(+75,30 \%)$. Resultados similares foram verificados em relação à adiposidade central, segundo os quais os estados nutricionais de sobrepeso e obesidade declinaram 14,30\% e $8,35 \%$, respectivamente, e a normalidade tendeu a se elevar $(+33,5)$.

A Figura 2 apresenta a prevalência de sobrepeso e obesidade geral e central em mulheres idosas, divididas por nível socioeconômico. Em relação à adiposidade geral, verificou-se que o sobrepeso praticamente não foi alterado $(+0,6 \%)$, compara-se a categoria socioeconômica alta à baixa. Por sua vez, as condições de obesidade $(+21,0 \%)$ e normalidade $(-22,0 \%)$ modificaramse consideravelmente. Quando a adiposidade central foi investigada, verificou-se que a obesidade pouco foi alterada $(-1,68 \%)$ na comparação

Tabela 2. Freqüência relativa e absoluta (\%) de normalidade, sobrepeso e obesidade geral e central, dividida por faixas etárias, de mulheres idosas. Curitiba (PR), 2006.

\begin{tabular}{|c|c|c|c|c|c|c|}
\hline \multirow{2}{*}{ Anos de vida } & \multicolumn{3}{|c|}{ Geral } & \multicolumn{3}{|c|}{ Central } \\
\hline & Normal & Sobrepeso & Obesidade & Normal & Sobrepeso & Obesidade \\
\hline $60-64,9$ & $54(18,2)$ & $145(49,0)$ & $97(32,8)$ & $70(23,6)$ & $95(32,1)$ & $131(44,3)$ \\
\hline $65-69,9$ & $69(22,4)$ & $124(40,3)$ & $115(37,3)$ & $62(20,2)$ & $103(33,7)$ & $141(46,1)$ \\
\hline $70-74,9$ & $59(23,8)$ & $107(43,1)$ & $82(33,1)$ & $52(21,1)$ & $81(23,8)$ & $114(46,2)$ \\
\hline $75-79,9$ & $37(25,0)$ & $62(41,9)$ & $49(33,1)$ & $26(17,8)$ & $54(37,0)$ & $66(45,2)$ \\
\hline$>80$ & $22(31,9)$ & $27(39,1)$ & $20(29,0)$ & $22(31,9)$ & $19(27,5)$ & $28(40,6)$ \\
\hline
\end{tabular}

Tabela 3. Freqüência relativa e absoluta (\%) de normalidade, sobrepeso e obesidade geral e central de mulheres idosas, segundo nível socioeconômico. Curitiba (PR), 2006.

\begin{tabular}{|c|c|c|c|c|c|c|}
\hline \multirow{2}{*}{ Classe de NSE } & \multicolumn{3}{|c|}{ Geral } & \multicolumn{3}{|c|}{ Central } \\
\hline & Normal & Sobrepeso & Obesidade & Normal & Sobrepeso & Obesidade \\
\hline Alta & $6(28,6)$ & $9(42,9)$ & $6(28,6)$ & $7(33,3)$ & $4(19,0)$ & $10(47,6)$ \\
\hline Média & $55(22,9)$ & $108(45,0)$ & $77(32,1)$ & $51(21,5)$ & $92(38,8)$ & $94(39,7)$ \\
\hline Baixa & $180(22,3)$ & $347(43,1)$ & $279(34,6)$ & $173(21,5)$ & $256(31,8)$ & $377(46,8)$ \\
\hline
\end{tabular}

NSE: nível socieconômico. 
530 | C.F. BUZZACHERA et al.
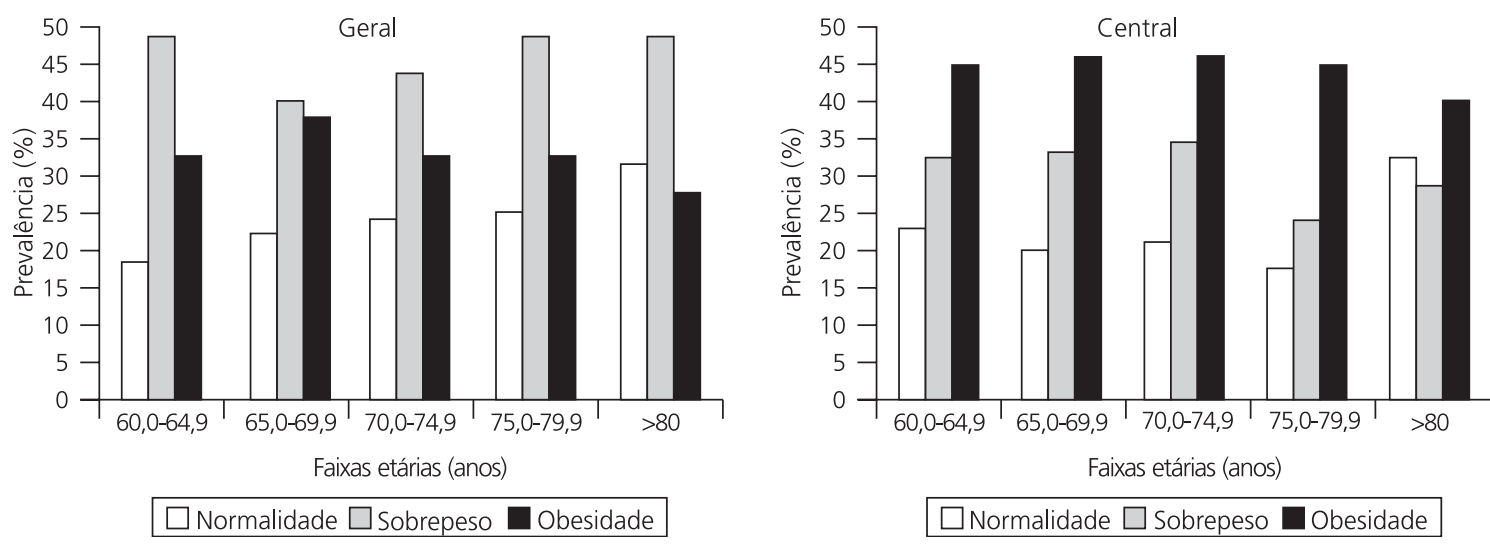

Figura 1. Classificação do estado nutricional de mulheres idosas, segundo faixas etárias. Curitiba (PR), 2006.
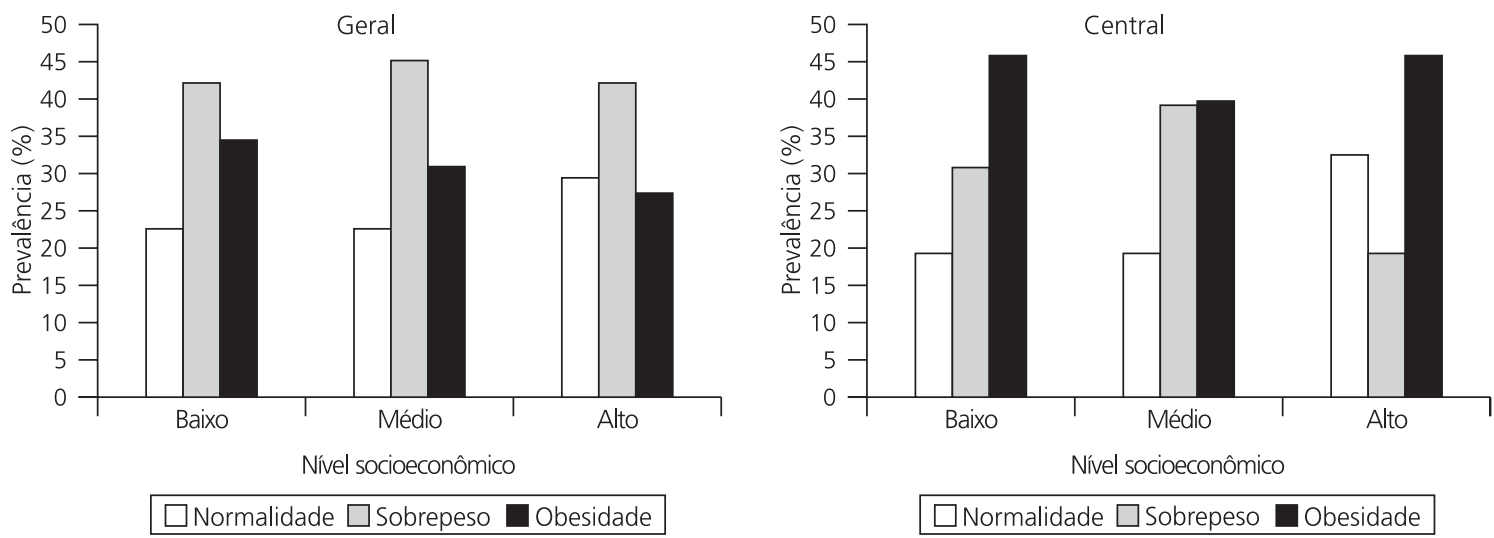

Figura 2. Classificação do estado nutricional de mulheres idosas, segundo nível socioeconômico. Curitiba (PR), 2006.

entre as categorias socioeconômicas alta e baixa, enquanto as classificações de normalidade $(-35,4 \%)$ e sobrepeso $(+67,3 \%)$ modificaram-se substancialmente.

\section{DISCUSS Ã O}

A elevada prevalência de sobrepeso e obesidade em idosos é considerada atualmente um problema de saúde pública global, principalmente devido ao fato de esse segmento populacional apresentar o maior crescimento nas últimas décadas em inúmeros países desenvolvidos e/ou em desenvolvimento ${ }^{7}$. Estudos anteriores demonstraram que essa condição de excessiva adiposidade corporal poderia representar uma elevação dos riscos à saúde, principalmente entre mulheres ${ }^{24}$ e idosas ${ }^{5,6}$. Infelizmente, pesquisas buscando investigar a prevalência desses fenômenos em idosos brasileiros são escassas ${ }^{5,6,24}$ Ainda, a investigação da excessiva adiposidade central nessa população é praticamente inexistente ${ }^{6}$.

A prevalência de sobrepeso $(42,7 \%)$ e obesidade $(33,1 \%)$ geral foi elevada entre as mulheres idosas. Estes valores são superiores aos apresentados em outros estudos nacionais ${ }^{5,6,24} \mathrm{e}$ internacionais ${ }^{25}$. Dados relativos ao Third National Health and Nutrition and Examination Survey (NHANES III), nos Estados Unidos, demonstraram que $64 \%$ das mulheres com idade entre 60-69 anos apresentaram $I M C \geq 25 \mathrm{~kg} / \mathrm{m}^{2,25}$. Contudo, valores superiores aos encontrados no presente 
estudo foram verificados por Gutiérrez-Fisac et al. ${ }^{8}$, cuja investigação identificou que cerca de $81 \%$ das mulheres espanholas com idade superior a 60 anos com $I M C \geq 25 \mathrm{~kg} / \mathrm{m}^{2}$. Esses estudos sugerem que diferenças ambientais poderiam influenciar a prevalência dos diferentes estados nutricionais entre os idosos.

Apesar de os resultados demonstrarem uma elevada prevalência de adiposidade corporal geral, uma análise cautelosa torna-se necessária. O valor médio de IMC foi $28,5 \mathrm{~kg} / \mathrm{m}^{2}$, denotando o estado nutricional de sobrepeso geral. Contudo, conforme proposto por James ${ }^{26}$, mulheres idosas apresentando valores de IMC entre 27 ou $28 \mathrm{~kg} / \mathrm{m}^{2}$ poderiam ser consideradas normais. Ainda, em estudo de acompanhamento conduzido por Mattila et al. ${ }^{27}$ envolvendo mulheres idosas, uma relação inversa entre IMC e mortalidade foi observada. Sujeitos com valores de $\mathrm{IMC}>28 \mathrm{~kg} / \mathrm{m}^{2}$ demonstraram uma taxa de sobrevivência relativa de 1,5 , comparados àqueles apresentando $\mathrm{IMC}<20 \mathrm{~kg} / \mathrm{m}^{2}$, nos quais o valor correspondente foi apenas 0,6. Desse modo, essa condição de moderado sobrepeso geral poderia ser considerada um interessante fator de proteção entre as mulheres idosas ${ }^{27}$.

Tais evidências poderiam também ser verdadeiras em relação à adiposidade corporal central. Porém, estudos recentes demonstram uma relação direta entre CC e risco para morbidades e mortalidade por todas as causas e/ou doenças cardiovasculares em idosos ${ }^{14-17}$. Isso resulta, principalmente, do fato de a CC ser considerada um indicador válido do conteúdo de tecido adiposo visceral $^{13}$, a qual em excesso poderia representar um aumentado risco à saúde devido às suas ações hormonais ${ }^{28}$. Desse modo, os resultados deste estudo indicando que a obesidade central foi o estado nutricional predominante $(45,1 \%)$ entre as mulheres idosas deveria ser observado com atenção. Estudo realizado por Foucan et al. ${ }^{29}$, utilizando IMC e CC como instrumento para screening de fator de risco cardiovascular em mulheres de 18 a 74 anos, mostrou valores de sensibilidade e especificidade em níveis entre $52,0 \%$ e $70,0 \%$ para IMC e $55,0 \%$ e $80,0 \%$ para CC, respectivamente. Esses achados revelam que, com a idade, há uma modificação na habilidade discriminante de parâmetros como IMC e CC em identificar fatores de risco para doenças cardiovasculares, contudo a CC foi a medida com maior habilidade em identificar presença ou ausência de fatores de risco.

No presente estudo, um valor médio de CC de $87,1 \mathrm{~cm}$ foi verificado entre as mulheres idosas, corroborando os achados de outros estudos ${ }^{6,8,12}$. Conforme sugerido por Lean et al. ${ }^{22}$, sujeitos adultos apresentando CC superior a $80 \mathrm{~cm}$ necessitam de um controle para evitar o aumento da massa corporal, enquanto aqueles com CC superior a $88 \mathrm{~cm}$ devem reduzir a massa corporal, diminuindo assim os riscos à saúde. Novamente, devido ao fato de a obesidade central ser o estado nutricional predominante entre as mulheres idosas $(45,1 \%)$, estratégias de intervenção preconizando a redução do excesso de adiposidade corporal, incluindo diminuição na ingestão dietética e/ou aumento no gasto energético diário, são necessárias.

O nível socioeconômico é considerado um importante fator de risco para a obesidade em sociedades em desenvolvimento ${ }^{4,30}$. No Brasil, estudos anteriores investigaram a relação entre essa variável e a obesidade ${ }^{3,4,30}$, porém nenhum deles analisou especificamente a população idosa. Desse modo, o presente estudo buscou estimar a prevalência de sobrepeso e obesidade geral e central em mulheres idosas, em relação à condição socioeconômica. O conhecimento da relação entre disparidades socioeconômicas e excesso de adiposidade corporal em diferentes segmentos etários é um tópico fundamental nas discussões de políticas de saúde pública.

Os resultados deste estudo demonstram que a maioria das mulheres idosas com $I M C \geq 25 \mathrm{~kg} / \mathrm{m}^{2}$ ou $\mathrm{CC} \geq 80 \mathrm{~cm}$ apresentam nível socioeconômico baixo. Estes resultados estariam de acordo com estudos recentes, nos quais se verificou que o segmento populacional de menor nível 
socioeconômico foi o que apresentou o mais rápido aumento no excesso de adiposidade corporal nas últimas décadas ${ }^{30}$. Contudo, a diferença entre a classificação de sobrepeso geral $(+0,60 \%)$ e obesidade central $(-1,68 \%)$, prevalentes na população idosa investigada, foi mínima na relação entre as categorias socioeconômicas alta e baixa. Monteiro et al. ${ }^{4}$ sugeriram que devido às modificações nos padrões de ingestão dietética e gasto energético ocorridas ultimamente, as prevalências de sobrepeso e obesidade na população adulta brasileira poderiam ser similares entre os diferentes NSE.

A prevalência de sobrepeso e obesidade geral e central declinou da primeira para a última faixa etária. Entretanto, conforme sugerido por Zamboni et al. ${ }^{28}$, em estudos envolvendo delineamento transversal, como verificado no presente estudo, esse declínio observado na prevalência de elevada adiposidade pode ser devido à mortalidade precoce ocorrida em mulheres apresentando excesso de gordura corporal em idades variando entre 50 e 70 anos. Desse modo, estudos com delineamento longitudinal são sugeridos para explicar adequadamente a associação entre a elevada adiposidade corporal geral e/ou central e a redução da longevidade.

Apesar de comumente empregado em estudos epidemiológicos envolvendo sujeitos idosos, o IMC não apresenta pontos de coorte para sobrepeso e obesidade geral específicos para essa população ${ }^{10}$. Modificações dependentes da idade ocorridas no denominador (estatura) e no numerador (massa corporal) do IMC poderiam resultar em estimativas inadequadas do verdadeiro estado nutricional da população idosa ${ }^{28}$. A necessidade do desenvolvimento de pontos de coorte de IMC específicos por idade de acordo com características demográficas tem sido recentemente sugerida ${ }^{10}$. A utilização conjunta da CC neste estudo teve o intuito de minimizar possíveis equívocos no diagnóstico geral do estado nutricional, devido ao emprego único do IMC. Por exemplo, verificou-se que, aproximadamente, $7,0 \%$ das mulheres idosas com um IMC inferior a
$25 \mathrm{~kg} / \mathrm{m}^{2}$ apresentaram uma circunferência de cintura superior a $80 \mathrm{~cm}$. Além disso, dentre as participantes com um IMC entre 25,0-29,9kg/ $\mathrm{m}^{2}$, cerca de $22,3 \%$ apresentavam uma CC superior a $88 \mathrm{~cm}$, denotando assim um estado nutricional de obesidade central.

Concluindo, verificou-se uma elevada prevalência de sobrepeso e obesidade geral e central em mulheres idosas em uma amostra populacional representativa do município de Curitiba (PR), independentemente da idade e do nível socioeconômico. A realização de futuros estudos envolvendo também a população idosa masculina é sugerida. Além disso, fica evidenciada a necessidade de programas públicos visando à prevenção e à redução do excesso de adiposidade corporal a partir de intervenção nos padrões de ingestão dietética e gasto energético, como a prática regular de exercício físico.

\section{COLABORADORES}

C.F. BUZZACHERA e M.P. KRAUSE participaram da idealização, do delineamento experimental, da coleta e análise dos dados, da redação e da revisão das versões do manuscrito até a versão final. H.M. ELSANGEDY, T. HALLAGE, P. GRANATO e K. KRINSKI participaram da coleta e da análise dos dados e da revisão das versões do manuscrito até a versão final. W. CAMPOS e S.G. SILVA participaram da idealização, da orientação, e da revisão de todas as versões do manuscrito até a versão final.

\section{REFERÊ NCIAS}

1. York DA, Rossner S, Caterson I, Chen CM, James WPT, Kumanyika $S$, et al. Obesity, a worldwide related to heart disease and stroke group I: worldwide demographics of obesity. Circulation. 2004; 110(18):e463-70.

2. Haslam DW, James WPT. Obesity. Lancet. 2005; 366(9492):1197-209.

3. Monteiro CA, Benicio MH, Conde W, Popkin B. Shifting obesity trends in Brazil. Eur J Clin Nutr. 2000; 54(4):342-6.

4. Monteiro CA, Conde WL, Popkin BM. The burden of disease from undernutrition and overnutrition in countries undergoing rapid nutrition transition: 
a view from Brazil. Am J Public Health. 2004; 94(3):433-4.

5. Tavares EL, Anjos LA. Perfil antropométrico da população idosa brasileira. Resultados da Pesquisa Nacional sobre Saúde e Nutrição. Cad Saúde Pública. 1999; 15(4):759-68.

6. Santos DM, Sichieri R. Índice de massa corporal e indicadores antropométricos de adiposidade em idosos. Rev Saude Publica. 2005; 38(2):163-8.

7. Camarano AA. O envelhecimento da população brasileira: uma contribuição demográfica. Rio de Janeiro: Instituto de Pesquisa e Estatística Aplicada; 2002.

8. Gutiérrez-Fisac JL, López E, Banegas JR, Graciani A, Rodriguez-Artelejo F. Prevalence of overweight and obesity in elderly people in Spain. Obes Res. 2004; 12(4):710-5.

9. Corrada MM, Kawas CH, Mozaffar F, Paganini-Hill A. Association of body mass index and weight change with all-cause mortality in the elderly. Am J Epidemiol. 2006; 163(10):938-49.

10. Cervi A, Franceschini SCC, Priore SE. Análise crítica do uso do índice de massa corporal para idosos. Rev Nutr. 2005; 18(6):765-75.

11. World Health Organization. Obesity: preventing and managing the global epidemic: Report of a WHO consultation. World Health Organ Tech Rep Ser. 2000; 894(1):1-253.

12. National Institute of Health. Clinical guidelines on the identification, evaluation, and treatment of overweight and obesity in adults: the evidence report. Obes Res. 1998: 6(2):S51-S210.

13. Janssen I, Heymsfield SB, Allison DB, Kotler DP, Ross R. Body mass index and waist circumference contribute to the prediction of nonabdominal, abdominal subcutaneous and visceral fat. Am J Clin Nutr. 2002, 75(4):683-8.

14. Sasaki JE, Krause MP, Gama MPR, Hallage T, Buzzachera CF, Dos Santos MG, et al. Influence of overall and abdominal adiposity on C-reactive protein levels in elderly women. Arq Bras Cardiol. 2007; 89(4):231-6.

15. Janssen I, Katzmarzyk PT, Ross R. Body mass index is inversely related to mortality in older people after adjustment to waist circumference. J Am Ger Soc. 2005; 53(12):2112-8.

16. Kanaya AM, Vittinghoff E, Shlipak MG, Resnick HE, Visser M, Grady D, et al. Association of total and central obesity with mortality in postmenopausal women with coronary heart disease. Am J Epidemiol. 2003; 158(12):1161-70.

17. Barbosa AR, Souza JPM, Lebrão ML, Laurenti R, Marucci MFN. Anthropometry of elderly residents in the city of São Paulo. Cad Saúde Pública. 2005: 21(6):1929-38.

18. Krause MP, Hallage T, Gama MPR, Sasaki JE, Miculis CP, Buzzachera CF, et al. Association between lipid profile and adiposity in women over age 60. Arq Bras Cardiol. 2007; 89(3):147-53.

19. Krause MP, Buzzachera CF, Hallage T, Pulner SB, DaSilva SG. Influência do nível de atividade física sobre a aptidão cardiorrespiratória em mulheres idosas. Rev Bras Med Esporte. 2007; 13(2):97-102.

20. Reijneveldt SA. Age in epidemiological analysis. J Epidemiol Comm Health. 2004; 57(3):397.

21. Lohman TG, Roche AF, Martorell R. Anthropometric standardization reference manual. Illinois: Human Kinetics Books; 1988.

22. Lean MEJ, Han TS, Morrison CE. Waist circumference as a measure for indicating need for weight management. BMJ. 1995; 311(6998): 158-61.

23. Associação Nacional de Empresas de Pesquisa. Critério de classificação socio-econômico. Rio de Janeiro: IBOPE; 1997.

24. Da Cruz IBM, Almeida MSC, Schwanke CHA, Moriguchi EH. Prevalência de obesidade em idosos longevos e sua associação com fatores de risco e morbidades cardiovasculares. Rev Assoc Med Bras. 2004; 50(2):172-7.

25. Flegal KM, Carroll MD, Kuczmarski RJ, Johnson $\mathrm{CL}$. Overweight and obesity in the United States: prevalence and trends, 1960-1994. Int J Obes. 1998; 22(1):39-47.

26. James WPT. Assessing obesity: are ethnic differences in body mass index and waist classification criteria justified. Obes Rev. 2005; 6(3):179-81.

27. Mattila K, Haavisto M, Rajala S. Body mass index and mortality in the elderly. BMJ. 1986; 292(6524):876-8.

28. Zamboni M, Mazalli G, Zoico E, Harris TB, Meigs $J B$, Di Francesco $V$, et al. Health consequences of obesity in the elderly: a review of four unresolved questions. Int J Obes. 2005; 29(9):1011-29.

29. Foucan L, Hanley J, Deloumeaux J, Suissa S. Body mass index (BMI) and waist circumference (WC) as screening tools for cardiovascular risk factors in Guadeloupean women. J Clin Epidemiol. 2002; 55(10):990-6.

30. Monteiro CA, Conde WL, Popkin BM. Incomespecific trends in obesity in Brazil: 1975-2003. Am J Public Health. 2007; 97(10):1808-12.

Recebido em: 2/2/2007

Versão final reapresentada em: 29/4/2008 Aprovado em: 28/5/2008 